\title{
Bearing Performance Degradation Assessment Using Lifting Wavelet Packet Symbolic Entropy and SVDD
}

\author{
Jianmin Zhou, Huijuan Guo, Long Zhang, Qingyao Xu, and Hui Li \\ School of Mechatronic Engineering, East China Jiaotong University, Nanchang 330013, China \\ Correspondence should be addressed to Jianmin Zhou; hotzjm@163.com
}

Received 16 June 2016; Accepted 28 September 2016

Academic Editor: Ganging Song

Copyright ( 2016 Jianmin Zhou et al. This is an open access article distributed under the Creative Commons Attribution License, which permits unrestricted use, distribution, and reproduction in any medium, provided the original work is properly cited.

\begin{abstract}
Bearing performance degradation assessment is of great significance for proactive maintenance and near-zero downtime. For this purpose, a novel assessment method is proposed based on lifting wavelet packet symbolic entropy (LWPSE) and support vector data description (SVDD). LWPSE is presented for feature extraction by jointing use of lifting wavelet packet transform and symbolic entropy. Firstly, the LWPSEs of bearing signals from normal bearing condition are extracted to train an SVDD model by fitting a tight hypersphere around normal samples. Then, the relative distance from the LWPSEs of testing signals to the hypersphere boundary is calculated as a quantitative index for bearing performance degradation assessment. The feasibility and efficiency of the proposed method were validated by the life-cycle data obtained from NASA's prognostics data repository and the comparison with Hidden Markov Model (HMM). Finally, the assessment results were verified by the envelope spectrum analysis method based on empirical mode decomposition and Hilbert envelope demodulation.
\end{abstract}

\section{Introduction}

Bearings play an important role in rotating machinery. The performance of bearings generally affects the operation reliability of whole equipment directly [1]. Actually, bearings always undergo a degenerative process from normal states to failure states. There is possibility to make some proper maintenance strategies to prevent the performance deterioration of bearings, if we can monitor the degenerative process of bearings in time [2]. Therefore, degradation assessment of bearings is significant to help reduce production downtime and save maintenance costs [3].

Vibration signals are always being used to monitor the running state of mechanical system because they carry a lot of information which indicates the health condition of mechanical equipment [4]. Based on vibration signal analysis, lots of fault diagnosis methods combining advanced signal processing and pattern recognition techniques have been proposed for bearings [5]. Although bearing fault diagnosis can be helpful for the indication of condition-based maintenance (CBM), they cannot reveal the degenerative trends of bearings. Given this problem, bearing performance degradation assessment has been a subject of extensive research in recent years, and more and more attention has been received for its benefits in implementing CBM strategies [6].

Generally speaking, performance degradation assessment of bearings mainly includes two steps, namely, feature extraction and degradation assessment. Until now, a lot of work covering these two aspects has been done. Conventional monitor indexes, such as root mean square (RMS) and kurtosis, have been frequently used as features for degradation assessment of bearings [7, 8]. Pan et al. proposed spectral entropy as a complementary index to evaluate the degradation state of bearings and the results of both simulations and experiments showed that spectral entropy can effectively reflect the degradation process of bearings [9]. Yu proposed a method of locality preserving projection for feature extraction and used a complementary index, namely, negative loglikelihood probability-based exponential weighted moving average statistic (NLLP-EWMA) to assess the performance degradation process of bearings [10]. Hong et al. proposed a method of wavelet packet-empirical mode decomposition for 
feature extraction and then used self-organization mapping (SOM) for performance degradation assessment of bearings [3]. Nelwamondo et al. utilized GMM and HMM to diagnose fault in rolling bearings, based on extracted features using Multiscale Fractal Dimension (MFD), Mel Frequency Cepstral Coefficients, and kurtosis. However, the major drawback of HMM classifier is that it is costly and complex [11]. Guo et al. investigated the Hilbert envelope spectrum and SVM method to diagnose REB with ball fault, inner race fault, and outer race fault [12]. The results show that the proposed method provides accurate diagnosis and good diagnostic resolution. Zhang et al. utilized particle swarm optimizationsupport vector machine (PSO-SVM) to realize the classification of fault location and degradation degree of rolling bearings [13]. Dong and Luo used principal component analysis (PCA) to reduce the dimension of original features and then constructed the LS-SVM model for degradation evaluation of bearings [14]. Sun et al. employed a kernel locality preserving projection-based method to obtain an index for evaluating the degradation degree of a bearing [15]. Liu et al. extracted zero crossing features of vibration signals and then employed a coupled Hidden Markov Model for assessing the performance degradation of bearings quantitatively [16]. Wang and Chen used bilateral spectrum for feature extraction as it could reveal nonlinearities and nonstationary characteristic and then employed a support vector data description (SVDD) model to assess the degradation process of bearings [17].

Though a lot of work has been done, there are still many challenges in assessing the performance degradation of bearings effectively. One of the challenges is how to extract more consistent features for degradation assessment since different features may only be useful at certain service stage or be applicable for specific degradation modes of bearings. For example, RMS can correlate well with the fault development of bearings but it is not sensitive to incipient faults, while Kurtosis Factor is sensitive to impulse faults (especially incipient faults) but it shows the poor stability as the damage grows $[7,8]$. Therefore, a composite index which can satisfy both the conditions of sensitivity and stability simultaneously is necessary. Another challenge is how to build an intelligent assessment model based on the extracted features.

To solve the abovementioned problems, a novel assessment method combining lifting wavelet packet symbolic entropy (LWPSE) and support vector data description (SVDD) is proposed in this paper. LWPSE is the combination of lifting wavelet packet transform (LWPT) and symbolic entropy. LWPT has some advantages over classical wavelet packet transform, including performing integer-tointeger wavelet transform and less computation and memory. Besides, LWPT can be reconstructed no matter how the prediction and update operators are designed. Recently, the applications of LWPT in fault diagnosis have achieved some good results [18, 19]. Symbolic time series analysis (STSA) provides a coarse grained description of a dynamical system based on a set of symbols. It is not sensitive to the measure noise, and hence it has a good robustness. Recently, it has been used for motoring degradation and fatigue damage using ultrasonic signals $[20,21]$. SVDD is a single value classification method developed from SVM

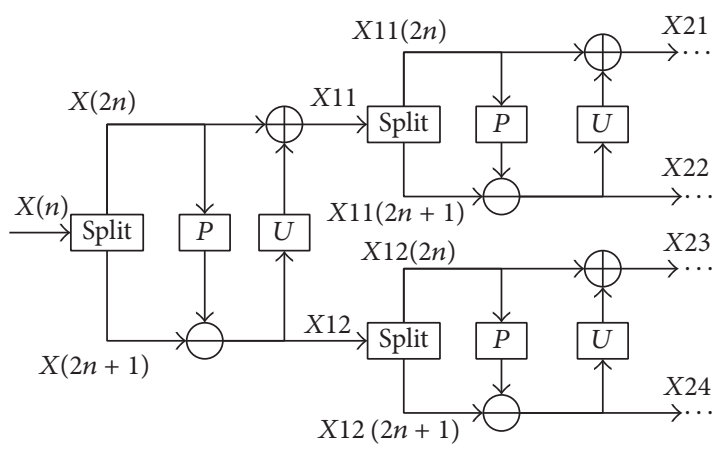

FIGURE 1: Block diagram of the forward transform of LWPT.

and the model can be established using normal data only. Moreover, it possesses the advantages of high computing efficiency and robustness. Therefore, SVDD has been widely used in degradation assessment of bearings [ $8,17,22]$.

The following paper is organized as follows. Sections 2 and 3 are dedicated to the theories of lifting wavelet packet symbolic entropy and SVDD, respectively. Section 4 presents the degradation assessment method based on LWPSE and SVDD and HMM. Experimental validation and the related analysis are provided in Section 5. The conclusions are provided in Section 6.

\section{Lifting Wavelet Packet Symbolic Entropy}

2.1. Lifting Wavelet Packet Transform. Lifting wavelet packet transform (LWPT) is realized through the lifting scheme. It no longer relies on Fourier transform, and all computations are implemented in time domain. Moreover, it is possible to obtain the wavelets with some special properties through the design of predictor and updater [23]. The forward transform process of LWPT is shown in Figure 1. We can run the lifting scheme backwards to derive the inverse transform from the forward transform. The process of lifting scheme mainly includes three steps: split, predict, and update. The detail procedure is as follows [24].

(1) Split. Split the original signal $X(n)=\{x(n), n=$ $0,1, \ldots, N\}$ into two subsets, namely, even samples $X(2 n)=$ $\{x(2 n), n=0,1, \ldots, N / 2\}$ and odd samples $X(2 n+1)=$ $\{x(2 n+1), n=0,1, \ldots, N / 2\}$, where $N$ is the length of $X(n)$.

(2) Predict and Update. In this step, each subband coefficient is calculated at level $j$ :

$$
\begin{gathered}
X_{j, 1}=X_{j-1,1}(2 n)+U\left(X_{j-1,1}(2 n+1)\right) \\
X_{j, 2}=X_{j-1,1}(2 n+1)-P\left(X_{j, 1}(2 n)\right)
\end{gathered}
$$

$$
\begin{gathered}
X_{j, 2^{j-1}}=X_{j-1,2^{j-1}}(2 n)+U\left(X_{j-1,2^{j-1}}(2 n+1)\right) \\
X_{j, 2^{j}}=X_{j-1,2^{j-1}}(2 n+1)-P\left(X_{j-1,2^{j-1}}(2 n)\right),
\end{gathered}
$$


where $P$ is a prediction operator and $U$ is an update operator. Here, $P$ and $U$ are designed by interpolation subdivision principle [25]. In the context of interpolation subdivision principle, the orders of $P$ and $U$ decide their values. Therefore, selecting different orders is equivalent to selecting different bilateral orthogonal wavelet filters with different vanishing moments. In the current study, the orders of $P$ and $U$ are both selected as 12 , since this can structure a wavelet function which is closely similar to mechanical impulses [24]. Then, we can obtain the values of $P$ and $U$.

2.2. Symbolic Entropy. Symbolic time series analysis (STSA) aims at symbolizing the time series data. The basic process of STSA is converting the original time series signals into sequences of discrete symbols via partition function, and then we can use statistical features of the symbols to describe the dynamic statuses of a system [26]. STSA includes the symbolization of original time series and the quantitative analysis of symbolic series.

Consider an original time series $X=\{x(i), i=$ $1,2, \ldots, N\}$. Converting $X$ into a binary symbol series $S=$ $\{s(k), k=1,2, \ldots, N\}$ is the most commonly used method in the symbolization of original time series. In this process, partitioning is the first step, and it is also a key step. Here, the partition function is obtained by finite difference method. It is defined as follows:

$$
s(k)= \begin{cases}0 & x(i+1)-x(i) \leq 0 \\ 1 & x(i+1)-x(i)>0,\end{cases}
$$

where $x(i)(i=1,2, \ldots, N)$ is the element of original time series signals $X$ and $s(k)$ is the binary symbol series which is equivalent to 0 or 1 . After the symbolization, a binary-coded symbol series $S$ that only contains "0" and "1" can be got. And the next thing to do is dividing $S$ into decimal sequences with length $L$, namely, words. When the delay time $T$ and the length of word $L$ are given, $S$ can be cut into short symbol sequences:

$$
\begin{array}{r}
P(k)=(s(k), s(k+T), \ldots, s(k+(L-1) T)), \\
k=1,2, \ldots, N-(L-1) T,
\end{array}
$$

where $N$ is the length of $S$. Then, $P(k)$ needs to be transformed to the word $D(k)$.

$$
\begin{aligned}
& D(k)=\sum_{j=1}^{L} 2^{L-j} P_{j}(k) \\
& P_{j}(k)=s(k+(j-1) T) .
\end{aligned}
$$

Based on the work above, a series of words can be obtained. Here, in order to reveal the intrinsic complexity of the original time series, the histogram of symbol sequence as the basis for quantitative statistics is introduced to measure the occurrence number of each word in all the words [27]. For example, a series of words derived from a binary-coded symbol series $S$ (the length of $S$ is 124 ) are $0,1,2, \ldots, 7$, and the occurrence numbers of each word are $27,16,2,18,15,4$,

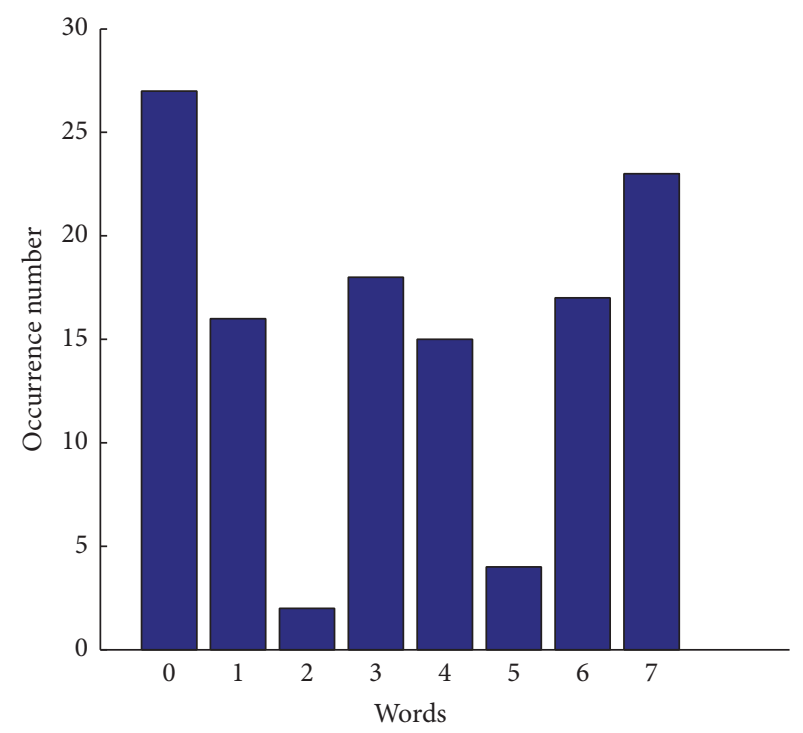

Figure 2: Histogram of symbol sequence word.

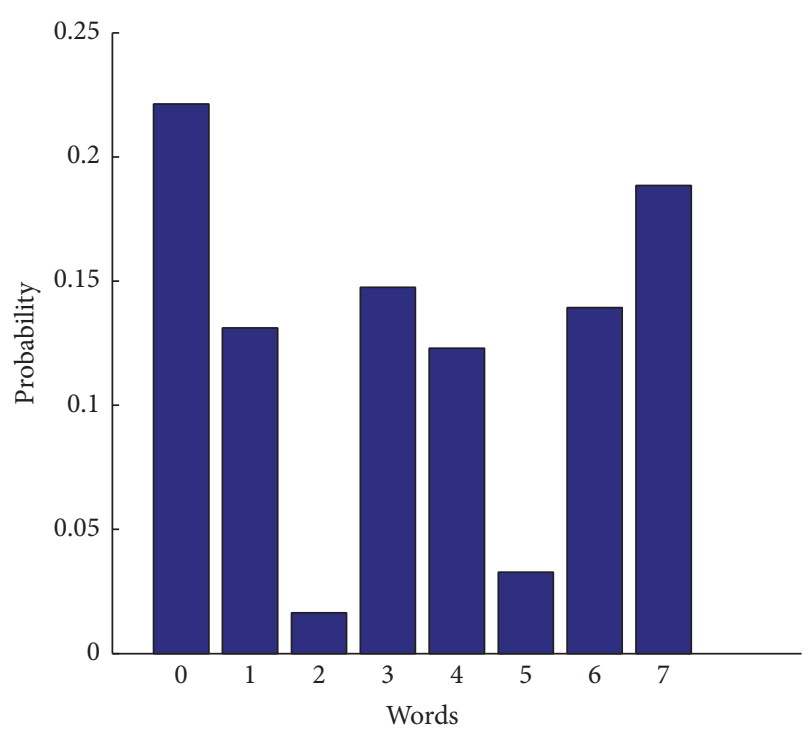

FIGURE 3: Probability of each word.

and 17; then, the histogram of symbol sequence can be shown in Figure 2, and the probability of each word (the normalization of Figure 2) can be shown in Figure 3. From the histogram of symbol sequence, we can use the modified Shannon entropy to describe the complexity of symbol sequence. The modified Shannon entropy is defined as

$$
H(T, L)=-\frac{1}{\log M} \sum p_{i} \log p_{i},
$$

where $p_{i}$ is the probability of the $i$ th word and $M$ is the number of all the symbol sequences. In the end, symbolic entropy is equivalent to the modified Shannon entropy of STSA.

Symbolic entropy is a complexity measurement; it can measure the complexity of signals. The vibration signals will 


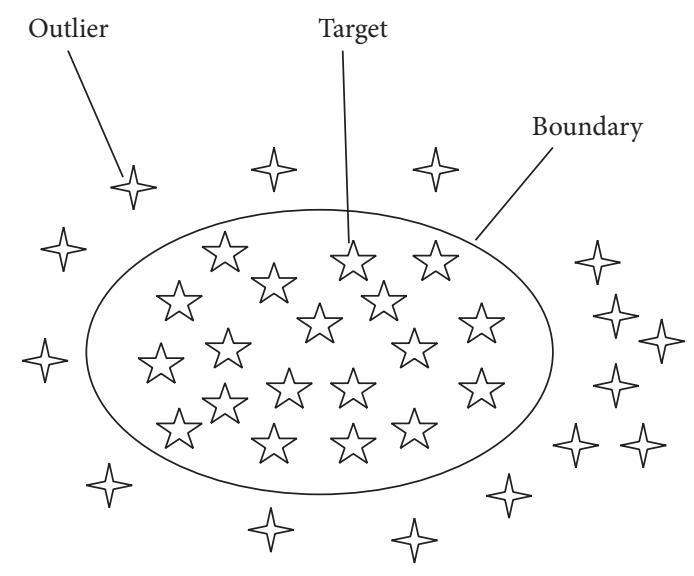

FIgURE 4: Schematic of the two-dimensional SVDD.

be more and more complex with the increase of fault severity, thus causing the increase of the entropy. Therefore, symbolic entropy can reveal the complexity of vibration signals.

2.3. Feature Extraction Using Lifting Wavelet Packet Symbolic Entropy. The basic steps in lifting wavelet packet symbolic entropy (LWPSE) based feature extraction are as follows:

(1) Employ LWPT to decompose bearing vibration signals. It has been illustrated in Section 2.1 that the best choices of the orders of $P$ and $U$ are both 12 . And in order to describe the features of bearing vibration signals more subtly, the decomposition level is defined as 4 . Then, the decomposition coefficients can be obtained.

(2) Choose the decomposition coefficients of each junction in the last level to reconstruct the original signals. Then, we can obtain the reconstructed signals.

(3) Symbolize the reconstructed signals. Here, the values of $T$ and $L$ are suggested to be 5 and 15 based on a lot of trials since there is no theoretical rule to determine them. Then, the symbolization of each reconstructed signal can be done and the histogram of symbol sequence can be obtained.

(4) Compute symbolic entropy of each reconstructed signal by formula (5). Then, a 16-dimensional feature vector $V=\left[H_{1}, H_{2}, \ldots, H_{16}\right]$ of bearing vibration signals can be obtained.

\section{Support Vector Data Description}

Support vector data description (SVDD) proposed by Tax and Duin is inspired by the theory of support vector machine (SVM) proposed by Vapnik [28]. The main idea of SVDD is to find an optimal hypersphere with minimal volume containing all or most targets, as shown in Figure 4.

Consider a training set $\left\{x_{i}, i=1,2, \ldots, n\right\}, n$ is the total number of samples. We try to find the optimal hypersphere which contains all or most normal samples. This hypersphere is described by center $c$ and radius $R$ and satisfies the following optimization function:

$$
\begin{array}{cl}
\min & L(R, c, \xi)=R^{2}+C \sum_{i=1}^{n} \xi_{i} \\
\text { s.t. } & \left(x_{i}-c\right)^{T}\left(x_{i}-c\right) \leq R^{2}+\xi_{i} \\
& \xi_{i} \geq 0, i=1,2, \ldots, n,
\end{array}
$$

where $C$ is a penalty parameter which controls the tradeoff between the volume of hypersphere and errors and $\xi_{i}$ are slack variables which permit a few training data to be outside the hypersphere.

Generally speaking, (6) is solved by introducing Lagrange multipliers and it can be transformed into the following maximizing function $L$ with respect to the Lagrange multipliers $\alpha_{i}$ :

$$
\begin{aligned}
\max & L=\sum_{i=1}^{n} \alpha_{i} x_{i} \cdot x_{i}-\sum_{i, j=1}^{n} \alpha_{i} \alpha_{j} x_{i} \cdot x_{j} \\
\text { s.t. } & \sum_{i=1}^{n} \alpha_{i}=1, \quad 0 \leq \alpha_{i} \leq C \forall i .
\end{aligned}
$$

Since the data in the input space are not always linearly predicted, we introduce a kernel function $K\left(x_{i}, x_{j}\right)=\left(\Phi\left(x_{i}\right)\right.$. $\left.\Phi\left(x_{j}\right)\right)$ to replace the inner product $\left(x_{i} \cdot x_{j}\right)$, where $K$ is a Mercer kernel. The kernel function $K\left(x_{i}, x_{j}\right)$ can map the data into a high-dimensional feature space and transform the nonlinear problem to a linear model. Any function meeting Mercer's theorem can be employed as kernel function, but not all of them are useful for SVDD. Gaussian kernel is the most commonly used function. It is defined as follows:

$$
K\left(x_{i}, x_{j}\right)=\exp \left(-\frac{\left\|x_{i}-x_{j}\right\|^{2}}{2 \sigma^{2}}\right),
$$

where $\sigma$ is the width parameter. Since Gaussian kernel can restrain the growing distances for large feature spaces for describing the target data more compactly, we employ it as $K\left(x_{i}, x_{j}\right)$. Then, (7) becomes

$$
\max L=\sum_{i=1}^{n} \alpha_{i} K\left(x_{i}, x_{i}\right)-\sum_{i, j=1}^{n} \alpha_{i} \alpha_{j} K\left(x_{i}, x_{j}\right) .
$$

All $\alpha_{i}$ are got by solving (9) and only a few of them are nonzero. The samples with $\alpha_{i}>0$ are called support vectors. Then, the radius $R$ is obtained by any support vector $x_{\mathrm{sv}}$ :

$$
\begin{aligned}
R^{2}= & K\left(x_{\mathrm{sv}} \cdot x_{\mathrm{sv}}\right)+\sum_{i, j=1}^{n} \alpha_{i} \alpha_{j} K\left(x_{i} \cdot x_{j}\right) \\
& -2 \sum_{i=1}^{n} \alpha_{i} K\left(x_{i} \cdot x_{\mathrm{sv}}\right) .
\end{aligned}
$$




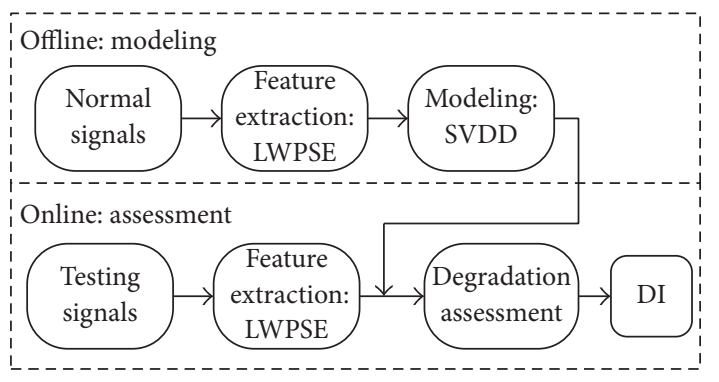

Figure 5: Procedure of performance degradation assessment.

For a new sample $x_{w}$, its distance to the center $c$ can be described as follows:

$$
\begin{aligned}
R_{w}^{2}= & K\left(x_{w} \cdot x_{w}\right)+\sum_{i, j=1}^{n} \alpha_{i} \alpha_{j} K\left(x_{i} \cdot x_{j}\right) \\
& -2 \sum_{i=1}^{n} \alpha_{i} K\left(x_{w} \cdot x_{i}\right) .
\end{aligned}
$$

Here, we introduce the theory of SVDD into bearing performance degradation assessment. Then, the relative distance between the new sample $x_{w}$ and the hypersphere boundary can be used as the degradation value (DV) of $x_{w}$. It is defined as follows:

$$
\mathrm{DV}=\frac{\left(R_{w}-R\right)}{R} .
$$

If $\mathrm{DV} \leq 0, x_{w}$ is accepted as a target which indicates that the bearing runs in a normal state. Otherwise, it is an outlier which indicates that the bearing runs in a degradation state.

\section{Degradation Assessment}

4.1. Degradation Assessment Based on LWPSE and SVDD. In this paper, we proposed a bearing performance degradation assessment method based on LWPSE and SVDD. The framework for performance degradation assessment is shown in Figure 5, which includes two parts, namely, offline modeling and online assessment. The steps of the proposed method are illustrated as follows:

(1) The historical normal signals of a rolling bearing are collected and their feature vectors are extracted using LWPSE.

(2) The feature vectors of normal state obtained from step (1) are used as training samples to establish an SVDD model. Then, the radius $R$ can be obtained by (10).

(3) As for the new testing signal $x_{w}$, its feature vectors are extracted using LWPSE. Then, the generalized distance is calculated using (11) which is related to the model established in step (2).

(4) The degradation value (DV) of $x_{w}$ is calculated using (12). Then, the degradation index with a series of DVs of testing signals can be obtained. Here, we set all the values of $\mathrm{DV}$ to 0 when $\mathrm{DV} \leq 0$.

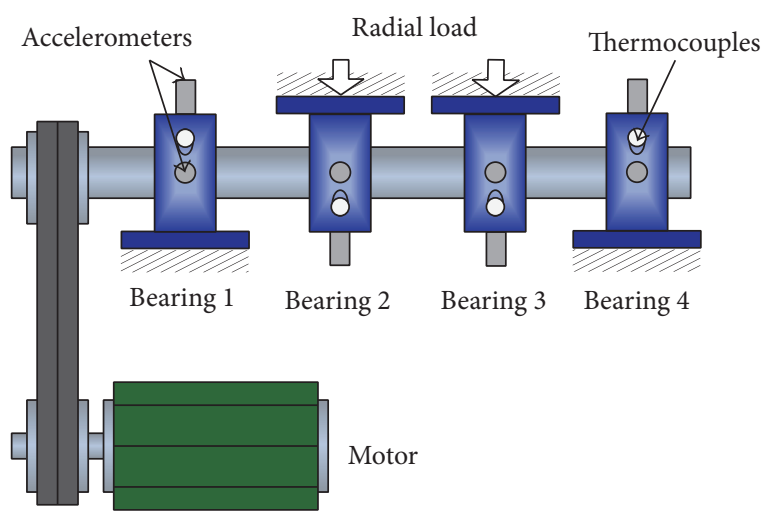

FIGURE 6: Bearing run-to-failure test rig.

From the degradation index, we can know that the bearing runs in a normal state while $\mathrm{DV} \leq 0$. Otherwise, it runs in a degradation state. Moreover, DV also reflects the degree of fault severity of a bearing, namely, the larger DV means the larger degree of fault severity.

\subsection{Degradation Assessment Based on Hidden Markov Model} (HMM). Hidden Markov model can be written as $\lambda=$ $(N, M, \pi, A, B)$ [29]. Where $N$ is the state number of Markov chain. Normal, initial failure, deterioration, deep degradation, and failure are the five states of bearings. $M$ is observation that may occur in each state. In the paper, $M$ is 11 and $\pi$ is the vector of initial probability distribution. $A$ is the model of the state transition probability matrix. Parameters $A$ and $\pi$ are generally uniform or selected randomly. $B$ is the model of observation probability matrix.

After model initialization is completed, this paper uses the first 200 sets of data to train model. It is set when two adjacent output of log-likelihood probability value are less than $10^{-3}$ and model training is completed. Then, enter the 986 group under testing data into the model trained above. When the program stops iterating, the likelihood probability output value of rolling bearing is got. Because the similar probability of their output is relatively small, this paper uses the $\log$-likelihood probability $\log P$ to represent the index of performance degradation. After getting its loglikelihood probability value, the paper uses plot function to draw performance degradation curve of rolling bearing.

\section{Experimental Validation}

5.1. Description of the Experiment. The experimental data were obtained from NASA's prognostics data repository [30]. The schematic of bearing run-to-failure test rig is shown in Figure 6. During the experiment, four Rexnord ZA-2115 double row bearings were tested on one shaft. The bearings have 16 rollers in each row. The roller diameter is $8.407 \mathrm{~mm}$. The pitch diameter is $71.501 \mathrm{~mm}$. And the tapered contact angle is $15.17^{\circ}$. The shaft was driven by a motor. The rotating speed was kept constant at $2000 \mathrm{rpm}$, and a $6000 \mathrm{lb}$ radial load was added to the shaft and bearings. All the bearings were lubricated. The test stopped when the debris adhered to 


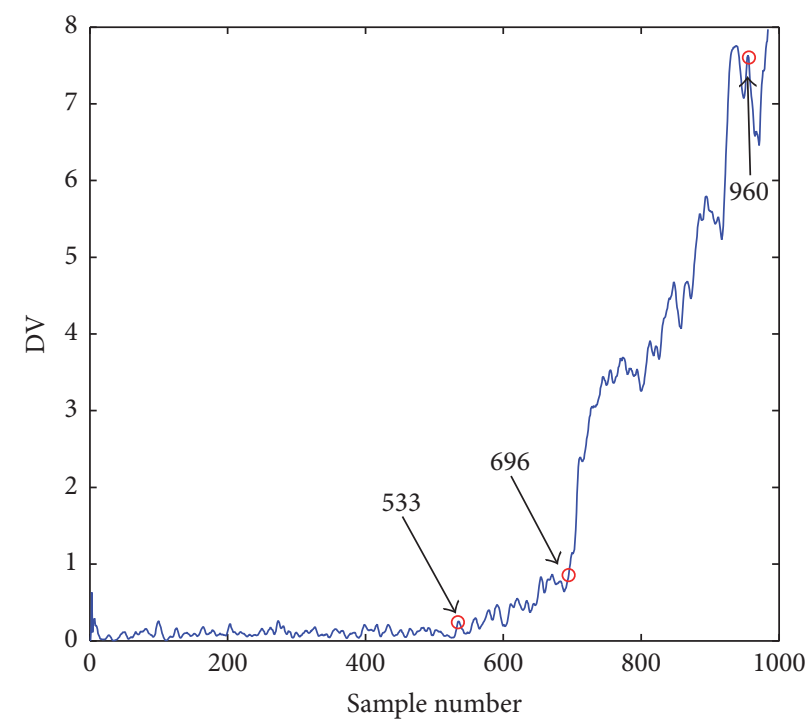

(a)

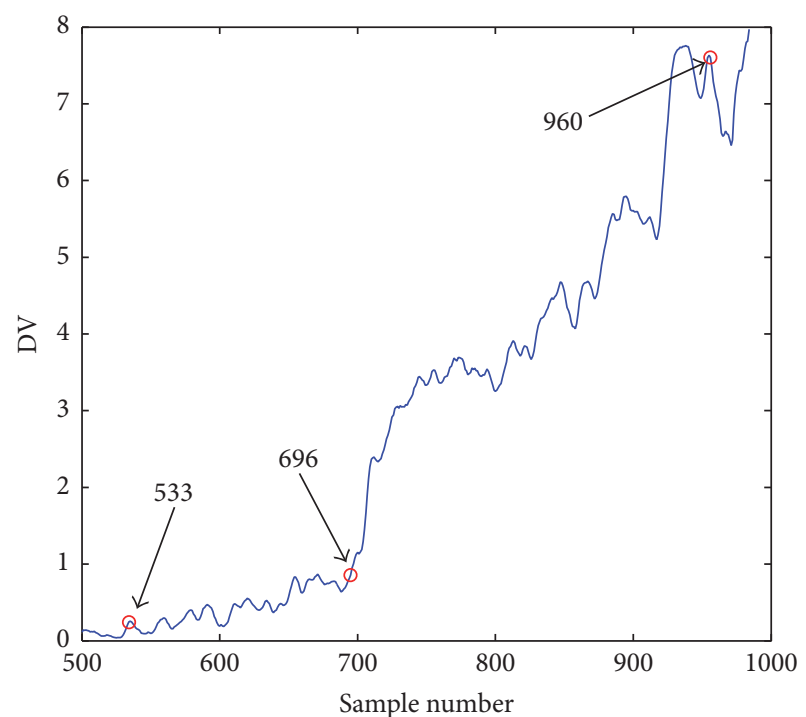

(b)

Figure 7: Assessment result using LWPSE and SVDD. (a) Life-cycle assessment result of bearing 1. (b) Local enlargement of (a).

the magnetic plug exceeded a certain level. On each bearing, two accelerometers PCB 353B33 were installed to collect the accelerations generated by the vibration signals, with a sampling rate of $20 \mathrm{kHz}$. The interval time of each collection was 10 minutes and the data length of each collection was 20480 points. The test was carried out for about 163 hours and it ended up with an outer race defect in bearing 1 . Therefore, the life-cycle data of bearing 1 was employed to assess the performance degradation of a bearing in this paper. From the geometric parameters of ZA-2115 bearings, we can know that its ball pass frequency of the outer race (BPFO) is about $236.4 \mathrm{~Hz}$.

\subsection{Experimental Results Analysis}

5.2.1. Assessment Results Using the Proposed Method. The first 200 sets of the life-cycle data of bearing 1 were used as normal signals, and their feature vectors were extracted using the feature extraction method proposed in Section 2.3. Then, we obtained a $200 * 16$ matrix, and the matrix was used to establish an SVDD model. After the model was established, all the life-cycle data were used as testing signals and their feature vectors were extracted. Then, the feature vectors were inputted to the trained SVDD model. Finally, the degradation index DV of bearing 1 was obtained. From the theory of time series analysis, we can know that to certain extent the output of a system at any time is determined by the output of the previous few moments. According to this theory, we can deal with the degradation index by five-point smoothing, namely, $\mathrm{DV}(t)=$ mean $(\mathrm{DV}(t-4: t))$, where mean mean $(\mathrm{DV})$ means getting mean values. The DV after five-point smoothing is shown in Figure 7. It is seen that the bearing's performance degradation process is clearly revealed by the DV. Before 5330 (the sample number is 533) minutes, the DV is approximately equal to 0 which indicates that the rolling bearing runs in the normal stage. At 5330 minutes, the DV has an obvious increase and it indicates that the initial fault begins to occur. After 6960 minutes, the increasing trend of the DV is more obvious and it indicates that the rolling bearing runs in the fault progression stage. After 9600 minutes, the DV has no more dramatic increase which indicates that the fault of rolling bearing is developing to failure. Also, sometimes the DV has some abrupt fluctuation since the edge of crack may be smoothed and rounded rapidly after their occurrence [31]. In sum, the performance degradation of bearing 1 could be divided into four periods successfully: normal stage from 0 to 5330 minutes, slight fault stage from 5330 to 6960 minutes, fault progression stage from 6960 to 9600 minutes, and developing-to-failure stage from 9600 minutes to the end.

In the proposed feature extraction method, there are two parameters to be predetermined, namely, the delay time $T$ and the length of word $L$ which is described in the third step of Section 2.3. Figure 8 plots the DV when the parameter $T$ is equal to different values. It can be seen that the increasing trend of the DV is the best when $T$ is equal to 5 as it can describe the degradation process more clearly. Figure 9 plots the DV when the parameter $L$ is equal to different values. It can be observed that the DV is the steadiest when $L$ is equal to 15 . Therefore, suitable $T$ and $L$ are meaningful for feature extraction.

5.2.2. Assessment Results Using RMS. RMS is one of the frequently used monitoring indexes. The RMS of bearing 1 is shown in Figure 10. It can be seen that the increasing trend of RMS amplitudes is not obvious before 6990 minutes which indicates the difference of RMS in the normal stage and slight fault stage is not evident, while the proposed degradation index is evident. In addition, it decreases after 7040 minutes which is not consistent with the degradation process. 


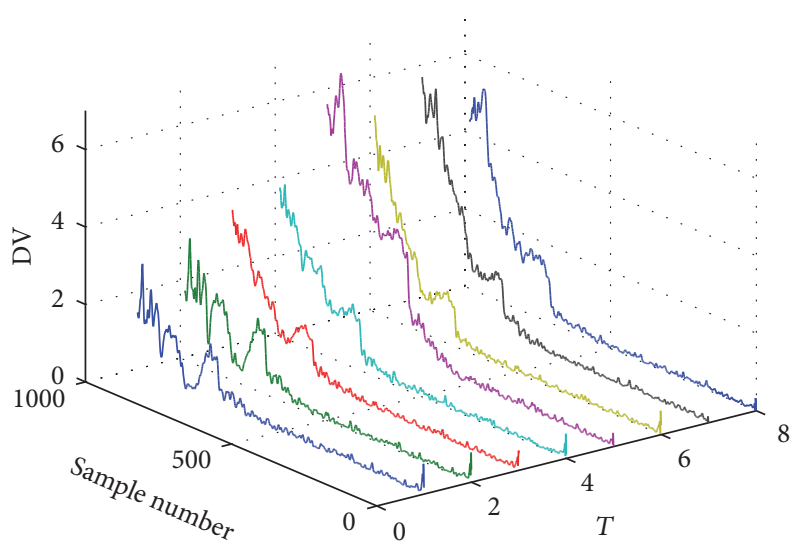

Figure 8: Assessment result with different values of $T$.

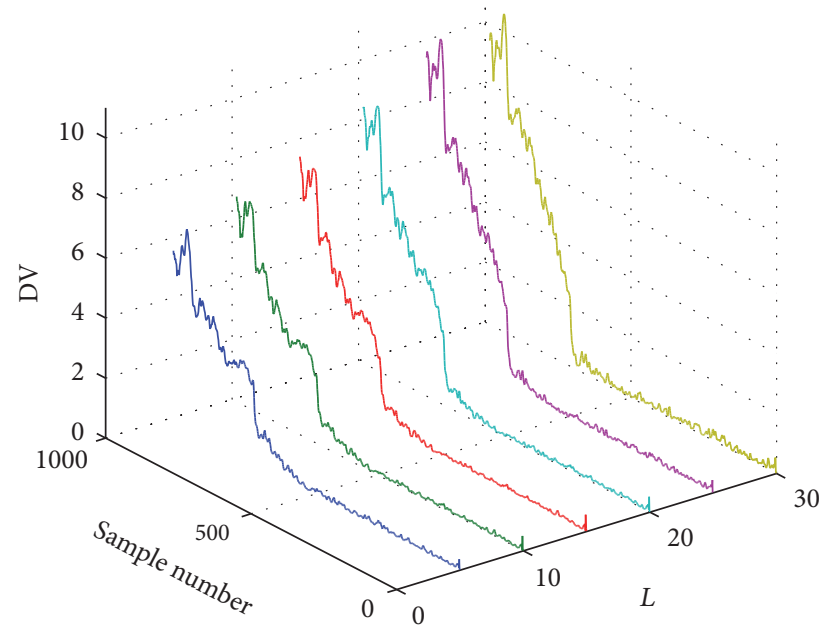

Figure 9: Assessment result with different values of $L$.

5.2.3. Assessment Results Using Hidden Markov Model. Performance degradation curve of the rolling bearing using HMM is shown in Figure 11.

The figure shows that probably in the 539th sample, the performance of the rolling bearing began to decline and early failure appeared. Probably in the 699th sample, the rolling bearing degraded. After the 820th sample, the rolling bearing exacerbated deeply. After the 963rd sample, the rolling bearing failed completely. We also can see that after about the 737th sample the performance degradation curve is inconsistent with its degree of fault. The overall trend of degradation curve is falling, but the curve still has relatively large fluctuations.

5.2.4. Assessment Results Using Lifting Wavelet Packet Entropy and $S V D D$. For a comparison between different feature extraction methods, the lifting wavelet packet entropy (LWPE) was used as feature to assess the performance degradation of bearing 1. LWPE is the combination of lifting wavelet packet transform and energy entropy. The basic steps in LWPE are similar to the steps in LWPSE, but

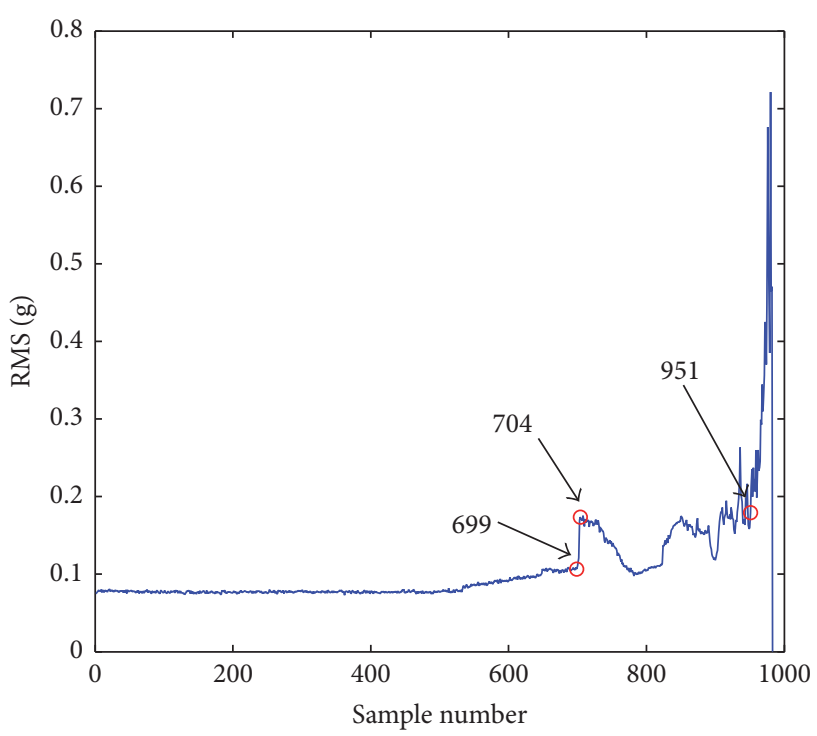

(a)

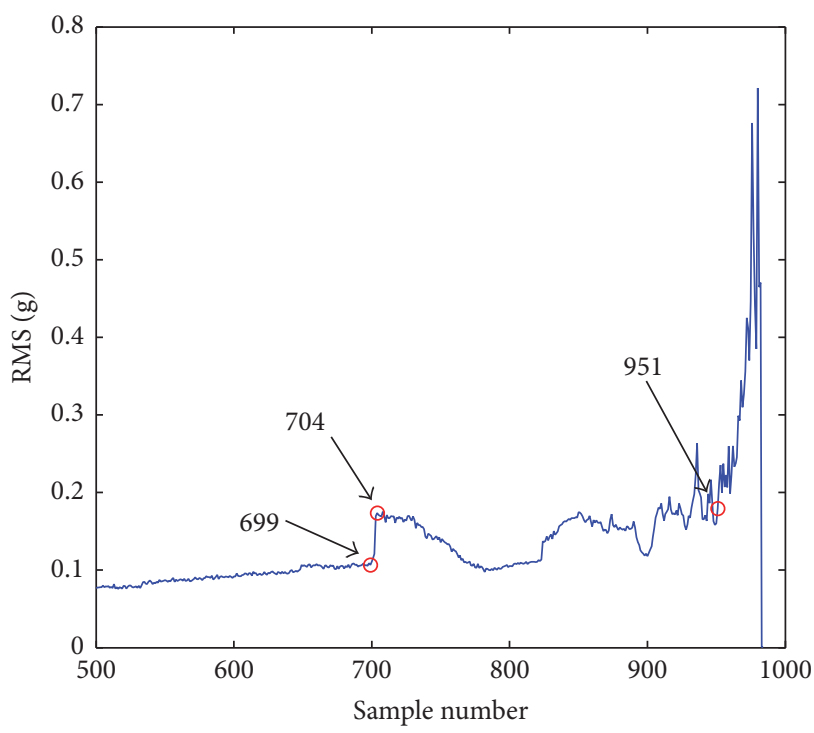

(b)

FIGURE 10: Root mean square (RMS). (a) Life-cycle RMS of bearing 1. (b) Local enlargement of (a).

LWPE extracts the energy entropy of each reconstructed signal obtained by lifting wavelet packet transform [32]. The assessment result based on LWPE and SVDD is shown in Figure 12. It is observed that the DV has an obvious increase at 5350 minutes and the increasing trend is more obvious after 7000 minutes, which indicates the description of normal stage and slight fault stage is similar to the assessment result based on LWPSE and SVDD. But the curve increases after 7930 minutes, which is not consistent with the degradation process. By comparison among Figures 7, 10, and 12, it is obvious that the degradation index proposed in this paper can reflect the bearing performance degradation process more effectively than the RMS and the degradation index based on LWPE and SVDD. 


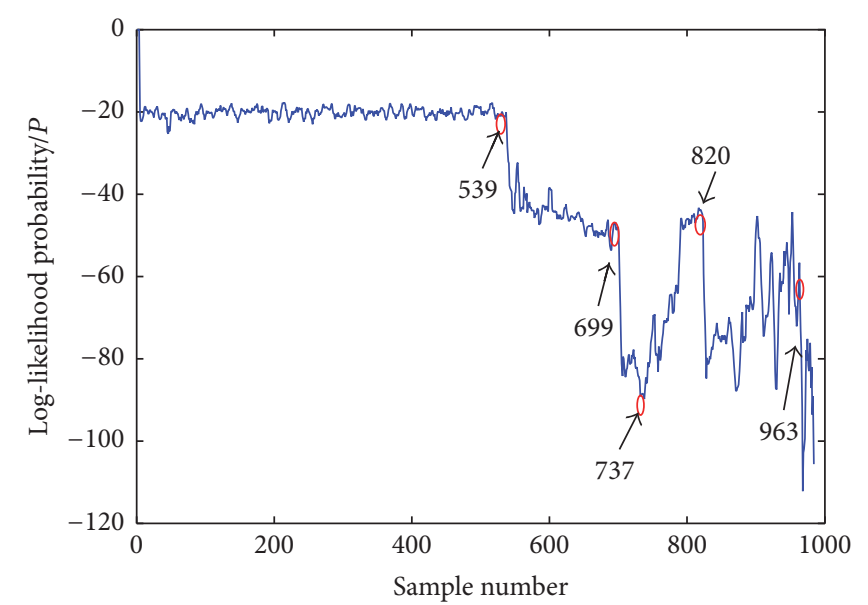

FIgURE 11: Assessment result using HMM.

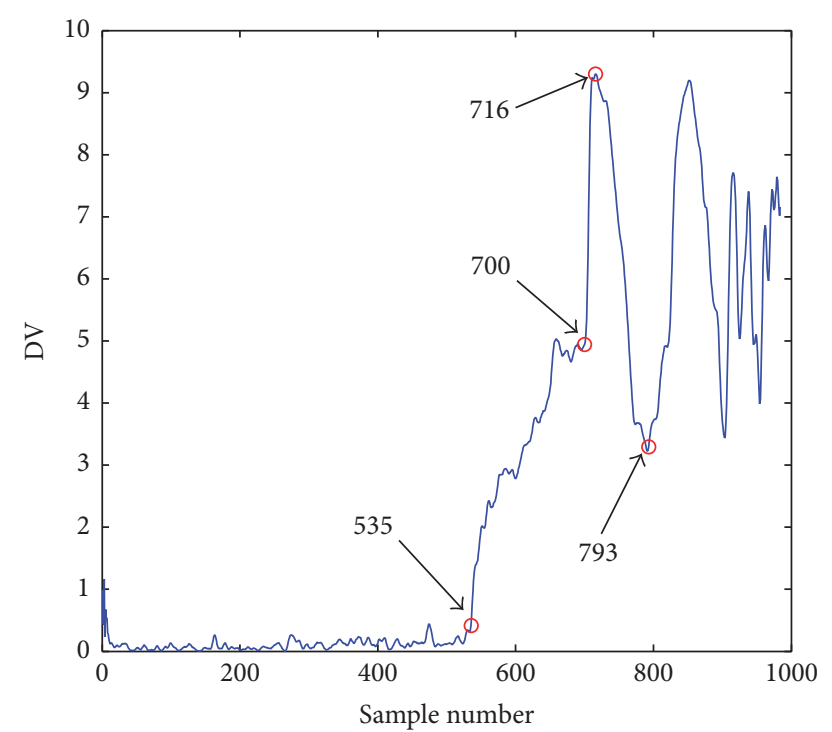

(a)

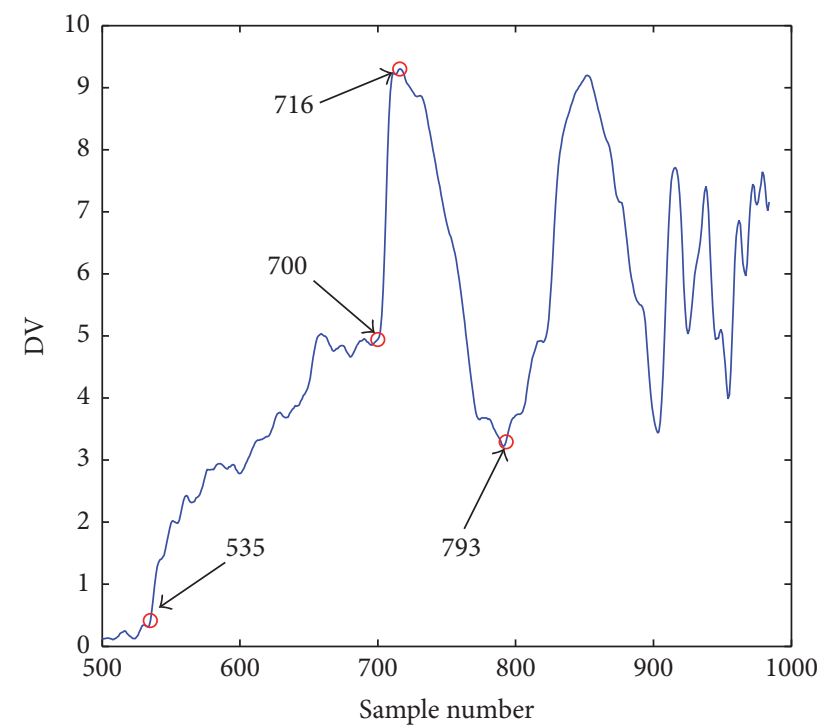

(b)

Figure 12: Assessment result using LWPE and SVDD. (a) Life-cycle assessment result of bearing 1. (b) Dramatic local enlargement of (a).

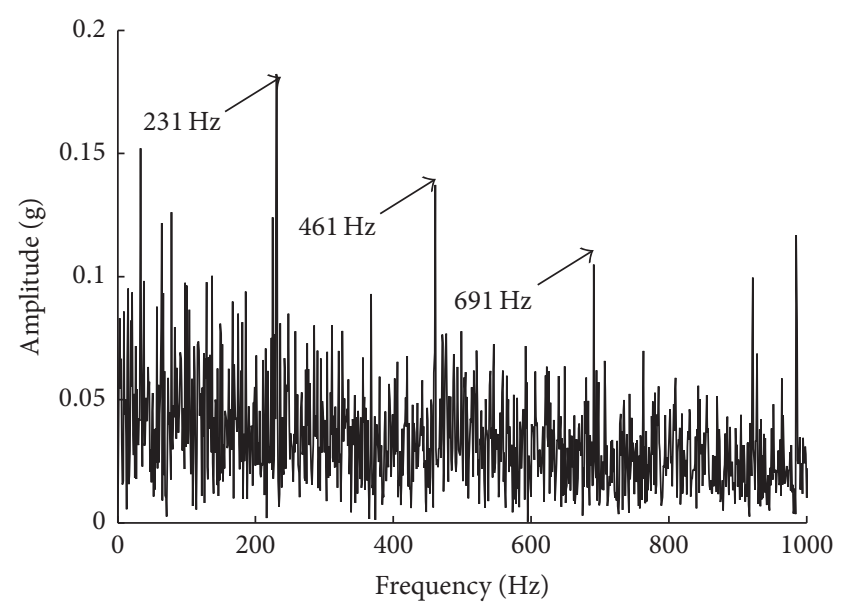

FIGURE 13: Demodulation result of the 533rd sample.

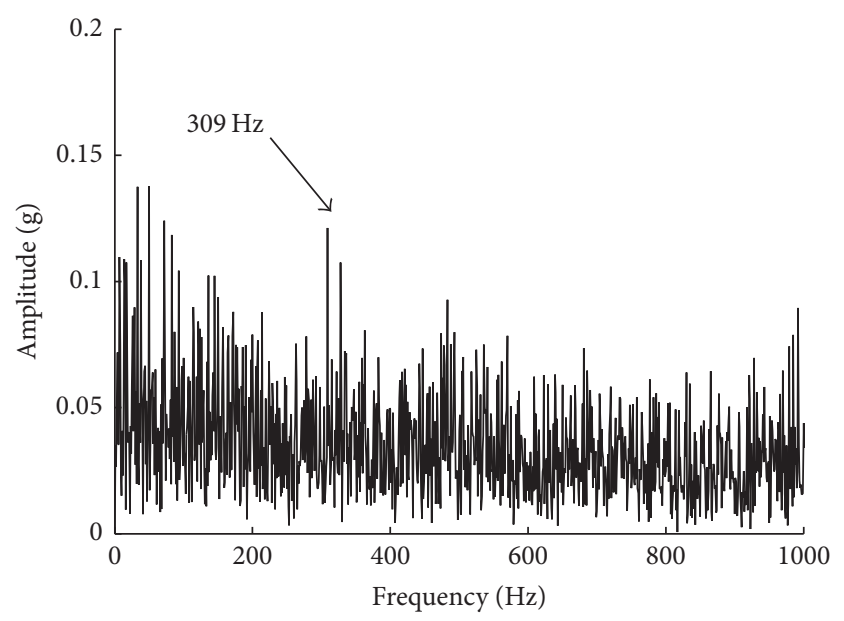

FIGURE 14: Demodulation result of the 532nd sample.

5.3. Correctness Validation of Assessment Results. To validate the correctness of assessment results that the initial fault occurs at 5330 minutes, the envelope spectrum analysis method based on empirical mode decomposition (EMD) and Hilbert envelope demodulation was employed in this paper. Here, we analyze the 533rd sample and the 986th sample. It was decomposed into several simple intrinsic mode functions (IMFs) by EMD first of all [33]. Then, we applied the Hilbert envelope demodulation analysis on IMF1 due to the fact that it is the highest frequency signal which includes the most detailed information of vibration signals [34]. The demodulation result is shown in Figure 13. It can be seen that there is an obvious spectrum peak at the frequency with $231 \mathrm{~Hz}$, which is close to the BPFO with $236.4 \mathrm{~Hz}$. Also, there exists obvious harmonic frequency characteristic. Moreover, the envelope spectrum of 532nd sample depicted in Figure 14 shows no obvious spectrum peak at the frequency close to the BPFO (the samples before the 532nd sample show the same results). The envelope spectrum of 986th sample depicted in Figure 15 shows that there is an obvious spectrum peak at the frequency with $231 \mathrm{~Hz}$, which is close to the BPFO 


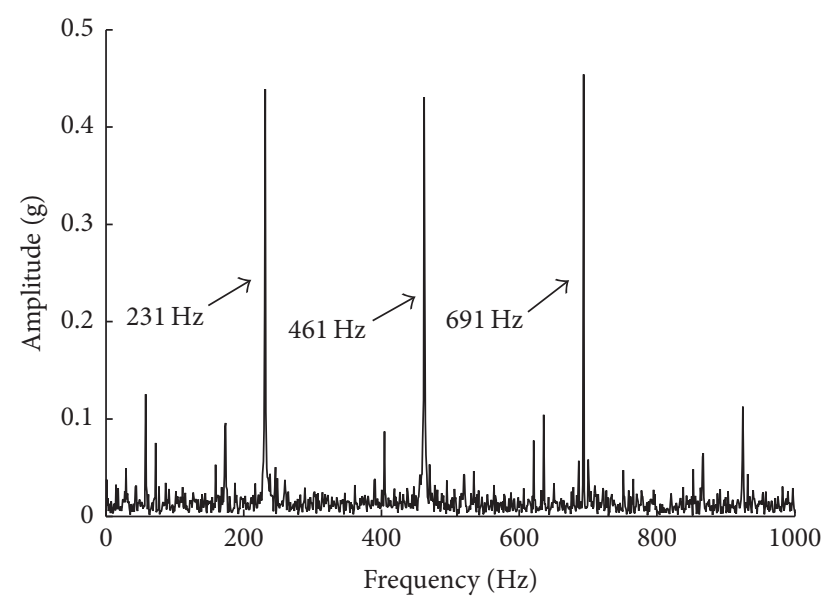

FIGURE 15: Demodulation result of the 986th sample.

with $236.4 \mathrm{~Hz}$. Also, there exists obvious harmonic frequency characteristic. It thus can be inferred that the initial fault of the outer race occurs at 5330 minutes. The analysis results are consistent with the previous assessment results.

\section{Conclusions}

In this paper, the degradation index based on LWPSE and SVDD is proposed for bearing performance degradation assessment. LWPSE is used to extract feature vectors and SVDD is employed to obtain the assessment results. The efficiency and validity of the proposed method are verified by the life-cycle data obtained from NASA's prognostics data repository. Analysis results show that, compared with the RMS and the degradation index based on LWPE and SVDD, the proposed degradation index is more sensitive to initial fault and it has a consistent increasing trend with the development of bearing fault. The method of HMM can show the overall performance degradation but its consistency of performance degradation is relatively poor. Further analysis shows that the degradation index is affected by the parameters $T$ and $L$ of the symbolic entropy; thus, suitable $T$ and $L$ are meaningful for feature extraction. Moreover, the correctness of assessment results is verified by the envelope spectrum analysis method based on EMD and Hilbert envelope demodulation. It may be concluded that the proposed method is better than HMM in the paper and it is of great significance in guiding the maintenance of rotating machinery.

\section{Competing Interests}

The authors declare that there is no conflict of interests regarding the publication of this paper.

\section{Acknowledgments}

This work was funded under the Natural Science Foundation of China, Grant no. 51205130. The authors are grateful to all study participants.

\section{References}

[1] W. B. Xiao, J. Chen, G. M. Dong, Y. Zhou, and Z. Y. Wang, "A multichannel fusion approach based on coupled hidden Markov models for rolling element bearing fault diagnosis," Proceedings of the Institution of Mechanical Engineers, Part C: Journal of Mechanical Engineering Science, vol. 226, no. 1, pp. 202-216, 2012.

[2] Y. N. Pan, J. Chen, and G. M. Dong, "A hybrid model for bearing performance degradation assessment based on support vector data description and fuzzy c-means," Proceedings of the Institution of Mechanical Engineers Part C: Journal of Mechanical Engineering Science, vol. 223, no. 11, pp. 2687-2695, 2009.

[3] S. Hong, Z. Zhou, E. Zio, and K. Hong, "Condition assessment for the performance degradation of bearing based on a combinatorial feature extraction method," Digital Signal Processing, vol. 27, no. 1, pp. 159-166, 2014.

[4] S. Hong, B. Wang, G. Li, and Q. Hong, "Performance degradation assessment for bearing based on ensemble empirical mode decomposition and gaussian mixture model," Journal of Vibration and Acoustics, vol. 136, no. 6, article 061006, 2014.

[5] I. El-Thalji and E. Jantunen, "A summary of fault modelling and predictive health monitoring of rolling element bearings," Mechanical Systems and Signal Processing, vol. 60, pp. 252-272, 2015.

[6] B. Zhang, L. Zhang, J. Xu, and P. Wang, "Performance degradation assessment of rolling element bearings based on an index combining SVD and information exergy," Entropy, vol. 16, no. 10, pp. 5400-5415, 2014.

[7] B. Tao, L. M. Zhu, H. Ding, and Y. Xiong, "An alternative time-domain index for condition monitoring of rolling element bearings-a comparison study," Reliability Engineering \& System Safety, vol. 92, no. 5, pp. 660-670, 2007.

[8] Z. Shen, Z. He, X. Chen, C. Sun, and Z. Liu, "A monotonic degradation assessment index of rolling bearings using fuzzy support vector data description and running time," Sensors, vol. 12, no. 8, pp. 10109-10135, 2012.

[9] Y. N. Pan, J. Chen, and X. L. Li, "Spectral entropy: a complementary index for rolling element bearing performance degradation assessment," Proceedings of the Institution of Mechanical Engineers, Part C: Journal of Mechanical Engineering Science, vol. 223, no. 5, pp. 1223-1231, 2009.

[10] J. B. Yu, "Local and nonlocal preserving projection for bearing defect classification and performance assessment," IEEE Transactions on Industrial Electronics, vol. 59, no. 5, pp. 2363-2376, 2012.

[11] F. V. Nelwamondo, T. Marwala, and U. Mahola, "Early classifications of bearing faults using hidden Markov models, Gaussian mixture models, mel-frequency cepstral coefficients and fractals," International Journal of Innovative Computing, Information \& Control, vol. 2, no. 6, pp. 1281-1299, 2006.

[12] L. Guo, J. Chen, and X. I. Li, "Rolling bearing fault classification based on envelope spectrum and support vector machine," Journal of Vibration and Control, vol. 15, no. 9, pp. 1349-1363, 2009.

[13] Y. Zhang, H. Zuo, and F. Bai, "Classification of fault location and performance degradation of a roller bearing," Measurement, vol. 46, no. 3, pp. 1178-1189, 2013.

[14] S. Dong and T. Luo, "Bearing degradation process prediction based on the PCA and optimized LS-SVM model," Measurement, vol. 46, no. 9, pp. 3143-3152, 2013. 
[15] C. Sun, Z. Zhang, Z. He, Z. Shen, B. Chen, and W. Xiao, "Novel method for bearing performance degradation assessment-a kernel locality preserving projection-based approach," Proceedings of the Institution of Mechanical Engineers, Part C: Journal of Mechanical Engineering Science, vol. 228, no. 3, pp. 548-560, 2014.

[16] T. Liu, J. Chen, and G. Dong, "Zero crossing and coupled hidden Markov model for a rolling bearing performance degradation assessment," Journal of Vibration and Control, vol. 20, no. 16, pp. 2487-2500, 2014.

[17] H. Wang and J. Chen, "Performance degradation assessment of rolling bearing based on bispectrum and support vector data description," Journal of Vibration and Control, vol. 20, no. 13, pp. 2032-2041, 2014.

[18] Y. Huang, C. Liu, X. F. Zha, and Y. Li, "An enhanced feature extraction model using lifting-based wavelet packet transform scheme and sampling-importance-resampling analysis," Mechanical Systems and Signal Processing, vol. 23, no. 8, pp. 2470-2487, 2009.

[19] Z. Wang, S. Bian, M. Lei, C. Zhao, Y. Liu, and Z. Zhao, "Feature extraction and classification of load dynamic characteristics based on lifting wavelet packet transform in power system load modeling," International Journal of Electrical Power and Energy Systems, vol. 62, pp. 353-363, 2014.

[20] R. A. Gupta, A. K. Wadhwani, and S. R. Kapoor, "Early estimation of faults in induction motors using symbolic dynamicbased analysis of stator current samples," IEEE Transactions on Energy Conversion, vol. 26, no. 1, pp. 102-114, 2011.

[21] D. S. Singh, S. Gupta, and A. Ray, "In-situ fatigue damage monitoring using symbolic dynamic filtering of ultrasonic signals," Proceedings of the Institution of Mechanical Engineers, Part G: Journal of Aerospace Engineering, vol. 223, no. 6, pp. 643653, 2009.

[22] X. Zhu, Y. Zhang, and Y. Zhu, "Bearing performance degradation assessment based on the rough support vector data description," Mechanical Systems and Signal Processing, vol. 34, no. 1-2, pp. 203-217, 2013.

[23] Y. N. Pan, J. Chen, and X. L. Li, "Bearing performance degradation assessment based on lifting wavelet packet decomposition and fuzzy C-means," Mechanical Systems and Signal Processing, vol. 24, no. 2, pp. 559-566, 2010.

[24] L. Zhang, G. L. Xiong, H. S. Liu, H. Zou, and W. Guo, "Fault diagnosis based on optimized node entropy using lifting wavelet packet transform and genetic algorithms," Proceedings of the Institution of Mechanical Engineers, Part I: Journal of Systems and Control Engineering, vol. 224, no. 5, pp. 557-573, 2010.

[25] W. Sweldens, "The lifting scheme: a custom-design construction of biorthogonal wavelets," Applied and Computational Harmonic Analysis, vol. 3, no. 2, pp. 186-200, 1996.

[26] R. Li, A. Mita, and J. Zhou, "Abnormal state detection of building structures based on symbolic time series analysis and negative selection," Structural Control and Health Monitoring, vol. 21, no. 1, pp. 80-97, 2014.

[27] T.-W. Chen and W.-D. Jin, "Feature extraction of radar emitter signals based on symbolic time series analysis," in Proceedings of the International Conference on Wavelet Analysis and Pattern Recognition (ICWAPR '07), pp. 1277-1282, Beijing, China, November 2007.

[28] D. M. J. Tax and R. P. W. Duin, "Support vector domain description," Pattern Recognition Letters, vol. 20, no. 11-13, pp. 1191-1199, 1999.
[29] L. Tao, C. Jin, and D. Guangming, "The rolling bearing fault diagnosis based on KPCA and coupled hidden Markov model," Vibration and Shock, vol. 21, pp. 85-89, 2014.

[30] J. Lee, H. Qiu, and G. Yu, "NASA Ames Prognostics Data Repository-Bearing Data Set," https://ti.arc.nasa.gov/ tech/dash/pcoe/prognostic-data-repository.

[31] R. Rubini and U. Meneghetti, "Application of the envelope and wavelet transform analyses for the diagnosis of incipient faults in ball bearings," Mechanical Systems and Signal Processing, vol. 15 , no. 2, pp. 287-302, 2001.

[32] W.-H. Li, B.-X. Dai, and S.-H. Zhang, "Bearing performance degradation assessment based on Wavelet packet entropy and Gaussian mixture model," Journal of Vibration and Shock, vol. 32, no. 21, pp. 35-40, 2013.

[33] J. B. Ali, N. Fnaiech, L. Saidi, B. Chebel-Morello, and F. Fnaiech, "Application of empirical mode decomposition and artificial neural network for automatic bearing fault diagnosis based on vibration signals," Applied Acoustics, vol. 89, pp. 16-27, 2015.

[34] J. Ma, J. Wu, Y. Fan, and X. Wang, "The rolling bearing fault feature extraction based on the LMD and envelope demodulation," Mathematical Problems in Engineering, vol. 2015, Article ID 429185, 13 pages, 2015. 


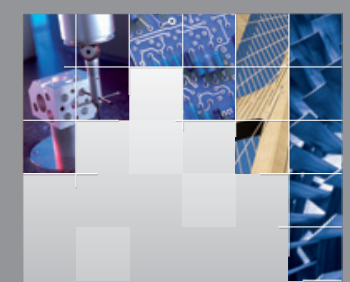

\section{Enfincering}
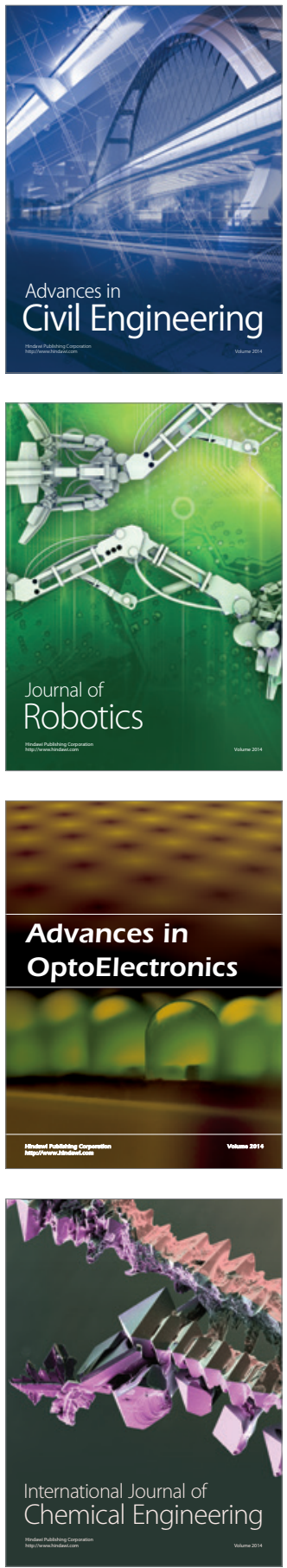

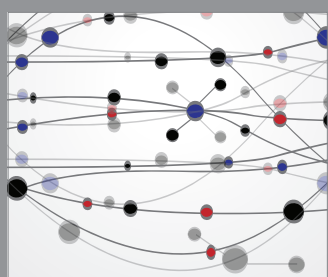

The Scientific World Journal

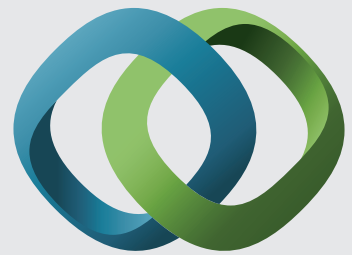

\section{Hindawi}

Submit your manuscripts at

http://www.hindawi.com
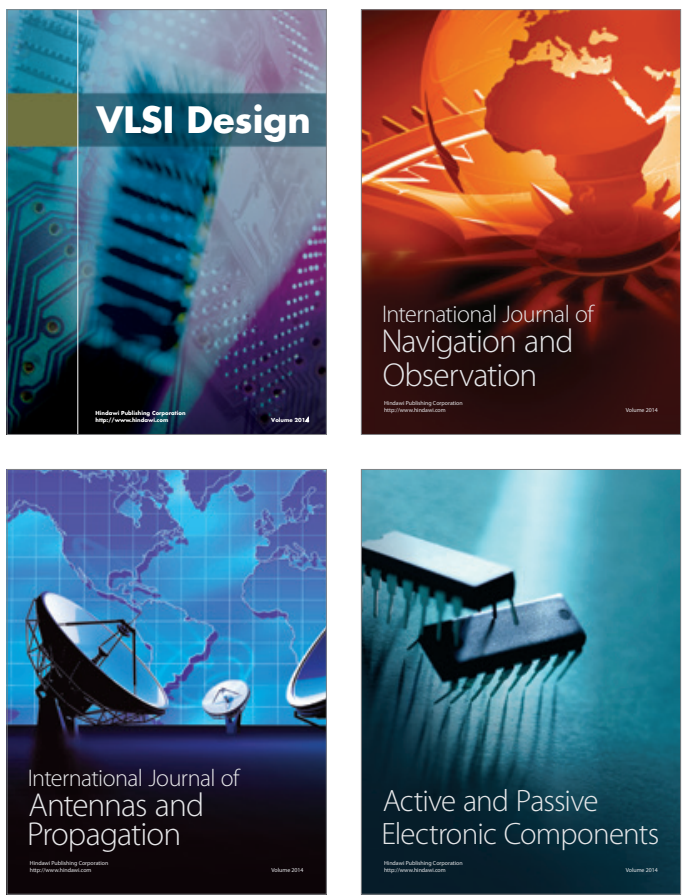
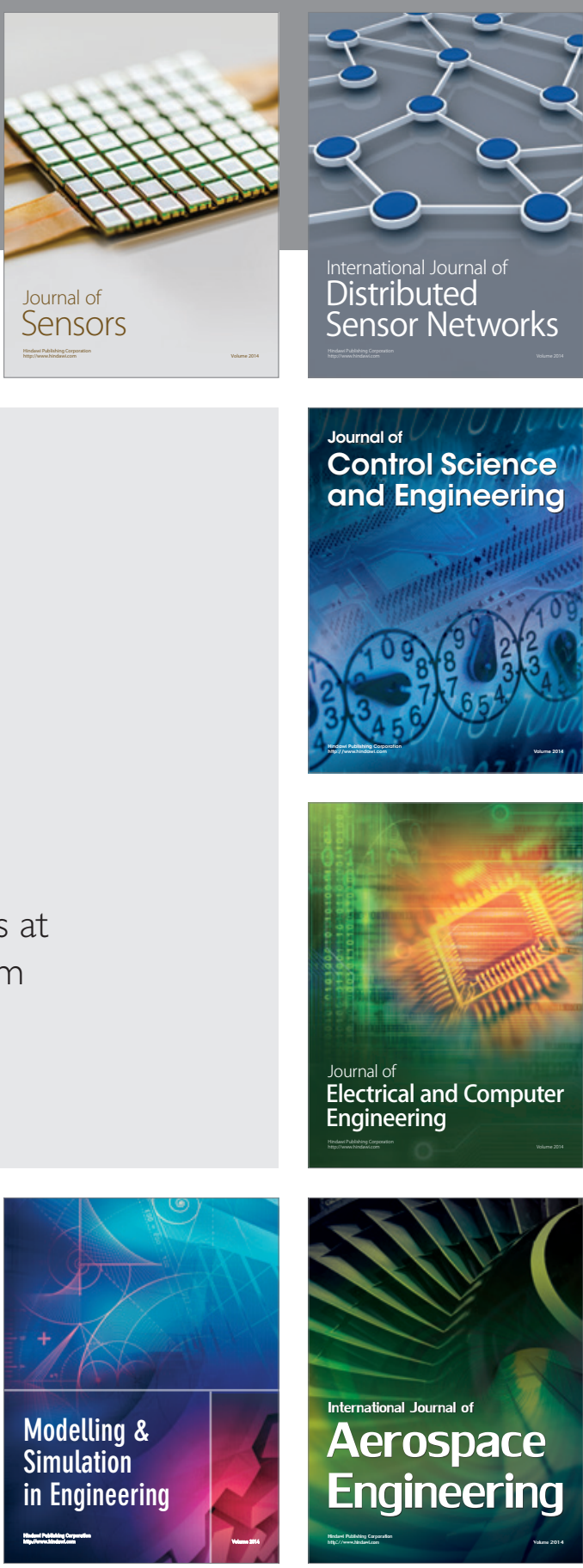

International Journal of

Distributed

Sensor Networks

Journal of

Control Science

and Engineering
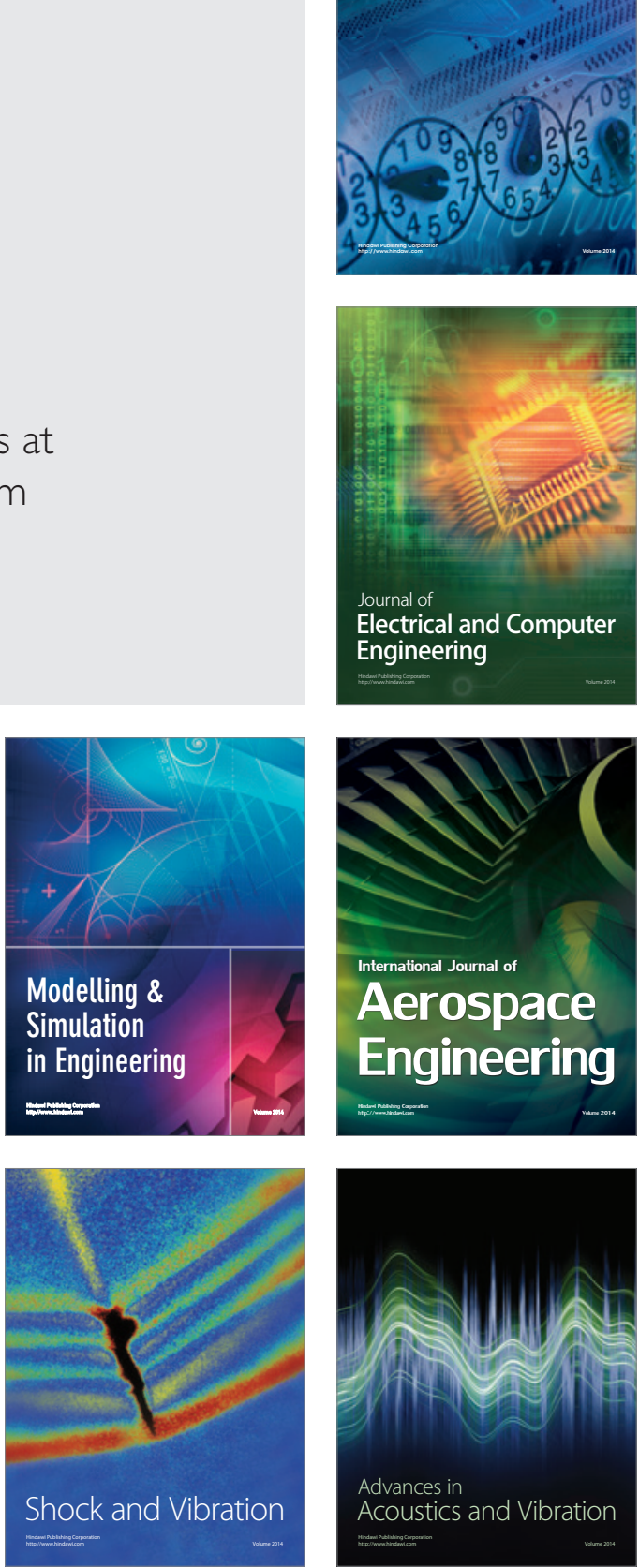